ИЗВЕСТИЯ АКАДЕМИИ НАУҚ ЭСТОНСКОИ ССР. ФИЗИКА * МАТЕМАТИКА

PROCEEDINGS OF THE ACADEMY OF SCIENCES OF THE ESTONIAN SSR.

PHYSICS * MATHEMATICS

1984, 33. 3

T. TOMCOH

\title{
СПЕКТР ВХОДНОГО ТОКА ГРУППЫ УПРАВЛЯЕМЫХ ВЫПРЯМИТЕЛЕЙ
}

\author{
(Представил Н. Эпик)
}

\section{Введение}

Представляет интерес изучение свойств системы управляемых выпрямителей на общих элементах $\left[{ }^{1}\right]$ как комплексной нагрузки для сети. Поскольку данная система рассматривается как система электропитания многопостовых электротехнологических негатронов, в частности как система электропитания группы плазмотронов постоянного тока, то это накладывает на названный вопрос специфические условия. Плазмотрон ввиду падающей вольт-амперной характеристики $\left[{ }^{2}\right]$ питается от выпрямителя, работающего в режиме генератора тока с постоянным выходным током $I_{d}=$ const. При этом выходное напряжение $U_{d}$ и угол управления $\alpha$ не постоянны. Они могут быть приняты как случайные величины, например, с нормальным распределением плотности вероятности вокруг заданной рабочей точки, однако они могут быть рассмотрены и как детерминированные величины, задаваемые конструктором или технологом: рабочая точка по напряжению может быть смещена выбором среднего газового расхода (для плазмотронов косвенного действия) и дополнительно расстоянием анода (для плазмотронов прямого действия). В настоящей работе за основу принят именно детерминированный подход, поскольку в этом случае наглядно выясняются основные законы входного тока. Постоянство выходного тока $I_{d}=$ const обеспечивается посредством как САР, так и индуктивного выходного фильтра $L_{d} \rightarrow \infty$ выпрямителя. Это обстоятельство позволяет интересующие нас процессы исследовать для нормализованного тока

$$
I_{d}^{*}=1=\text { const } \neq f(\alpha) .
$$

Под системой электропитания мы подразумеваем систему выпрямителей с общей диодной, а также с общей тиристорной частью [ $\left.{ }^{1}\right]$. Однако ради общности ниже рассматривается и параллельная работа системы 6-пульсных выпрямителей, которая раньше в научной литературе не анализировалась. Из вопросов, связанных с групповой работой, ранее рассмотрены лишь некоторые. Так, в $\left[{ }^{3}\right]$ рассматривается неидеальная компенсация $5,7,17$ и 19-й гармоник в каскаде из двух 6-пульсных выпрямителей, обусловленная неточностью СИФУ. В $\left[{ }^{4,5}\right]$ изучены вероятностные характеристики гармонических составляющих суммарного тока при случайном изменении сопротивления нагрузок 6-пульсных выпрямителей, входящих в систему. В [ $\left.{ }^{6}\right]$ установлено, что в каскаде из двух 6-пульсных выпрямителей при несимметрическом управлении достигается гармонический состав, который дает ему преимущества перед 
одним 6-піульсным, но уступает 12-пульсному. Такие важные проблемы, как зависимость гармонического состава суммарного тока от числа входящих в систему выпрямителей, углов управления и разности углов управления, в литературе не освещены и составляют предмет настоящей работы.

Гармонический состав выходного тока полууправляемого моста в виде графической зависимости амплитуд гармоник $\varrho_{v}=f\left(U_{d}\right), \quad v \in$ $\in\{2,4,5,7\}$ приведен в $\left[{ }^{7}\right]$, однако этого недостаточно для настоящего исследования. Гармонический состав входного тока 6-пульсного моста изучен хорошо с высокой точностью аппроксимации $\left[{ }^{8-13}\right]$, но полученные результаты не применимы ввиду громоздкости их и зачастую могут быть интерпретированы лишь по графикам аналитических функций. Поэтому нами использована грубая аппроксимация входного тока с прямоугольным импульсом, что соответствует нулевому углу коммутации $\gamma=0$. Подобная аппроксимация общепринята $\left[{ }^{7,14}\right]$ и достаточна для выяснения основных закономерностей. Сопутствующие ошибки увеличиваются с ростом порядка гармоник, однако для сети существенны именно гармоники низких порядков, которые обладают большой энергией. В отличие от общепринятой методики $\left[{ }^{14}\right]$ в настоящей работе не может быть применена относительная система координат (начало отсчета $\omega t=0$ смещено центром импульса тока), так как в последнем случае мы потеряли бы фазовые соотношения, которые необходимо соблюдать при рассмотрении групповой работы.

Для упрощения задания в настоящей работе применялся метод наложения, однако в отличной от использованной в $\left[{ }^{15}\right]$ форме. Действующий в линейной цепи (сеть может быть рассмотрена как линейная цепь) неизвестный сигнал $f(\omega t)$ можно разложить на два сигнала

$$
f(\omega t)=\varphi(\omega t) \pm \Phi(\omega t),
$$

где $\varphi(\omega t)$ - известный сигнал (сигнал с известным спектром) и $\Phi(\omega t)$ - сигнал помех. Если $f(\omega t)$ есть периоднческая функция, которая удовлетворяет условиям Дирихле, то она может быть разложена следующим образом

$$
f(\omega t)=b_{0}+\sum_{v=1}^{\infty} a_{v} \sin v \omega t+\sum_{v=1}^{\infty} b_{v} \cos v \omega t
$$

где амплитуды гармонических составляющих

$$
\begin{gathered}
\sum_{v=1}^{\infty} a_{v} \sin v \omega t=\sum_{v=1}^{\infty}(1 / \pi) \sin v \omega t \int_{-\pi}^{+\pi} f(\omega t) \cos v \omega t d \omega t= \\
=\sum_{v=1}^{\infty}(1 / \pi) \sin v \omega t \int_{-\pi}^{+\pi}[\varphi(\omega t) \pm \Phi(\omega t)] \cos v \omega t d \omega t= \\
=\sum_{v=1}^{\infty}(1 / \pi) \sin v \omega t\left[\int_{-\pi}^{+\pi} \varphi(\omega t) \cos v \omega t d \omega t+\int_{-\pi}^{+\pi} \Phi(\omega t) \cos v \omega t d \omega t\right]= \\
=\sum_{v=1}^{\infty} a_{\varphi v} \sin v \omega t+\sum_{v=1}^{\infty} a_{\Phi v} \sin v \omega t .
\end{gathered}
$$

Аналогично можно показать, что

$$
\sum_{v=1}^{\infty} b_{v} \sin v \omega t=\sum_{v=1}^{\infty} b_{\varphi v} \cos v \omega t+\sum_{v=1}^{\infty} b_{\Phi v} \cos v \omega t,
$$

т. е. разложение искомой периодической функции может быть представлено как сумма разложений известной функции и сигнала помех. 
Рис. 1. Эпюры токовых сигналов для групп: $a-$ управляемого и неуправляемого 6-пульсных мостовых выпрямителей, 6 - полууправляемых мостовых выпрямителей, в - сигнала помехи и 2 - тиристорных регуляторов с общей тиристорной группой.
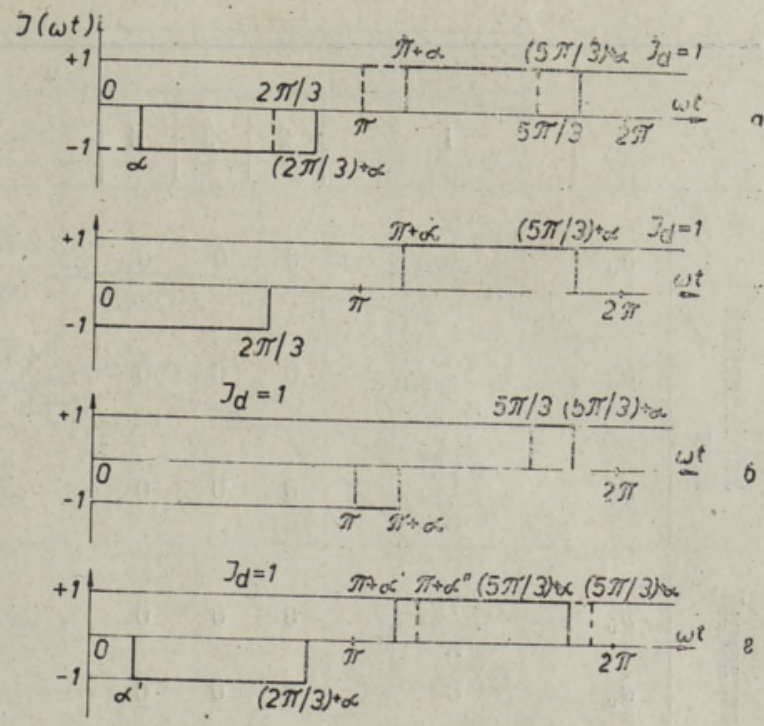

\section{Групповая работа 6-пульсных мостов}

Эпюра тока управляемого моста показана на рис. 1,a в системе координат, привязанной к точке естественной коммутации $\alpha=0$. Согласно вышесказанному,

$$
\begin{array}{llll}
I(\omega t)=-1 & \text { при } & \alpha<\omega t<2 \pi / 3+\alpha \quad \text { и } \\
I(\omega t)=1 & \text { при } & \pi+\alpha<\omega t<5 \pi / 3+\alpha .
\end{array}
$$

Коэффициенты разложжения Фурье

$$
\begin{aligned}
& a_{v}=(1 / \pi) \int_{0}^{2 \pi}(-\cos v \omega t+\cos v \omega t) d \omega t \\
& b_{v}=(1 / \pi) \int_{0}^{2 \pi}(-\sin v \omega t+\sin v \omega t) d \omega t
\end{aligned}
$$

показаны в табл. 1 . Приняв $\alpha=0$, получаем из (3) частный случай неуправляемого моста. В табл. 1 показаны также амплитуды соответствующих гармоник $\varrho_{v}(\alpha)=$ const, полученные из условия $I_{d}=$ const. Для единичного $l$-го выпрямителя соотношение амплитуды гармоники высшего порядка и амплитуды первой гармоники

$$
\zeta_{v l}=\mathrm{Q} v l / \mathrm{Q}_{1 l}=1 / v=\mathrm{const}
$$

инвариантно углу управления. Поскольку фильтрокомпенсирующие устройства высших гармонических составляющих в сети приходится проектировать для каждой гармоники отдельно, то в некоторых технических приложениях указанное соотношение характеризует состояние сети лучше, чем известный коэффициент несинусоидальности.

В системе из $n$ 6-пульсных выпрямителей с различающимися углами управления

$$
a_{v n}=\sum_{l=1}^{n} a_{v l}
$$




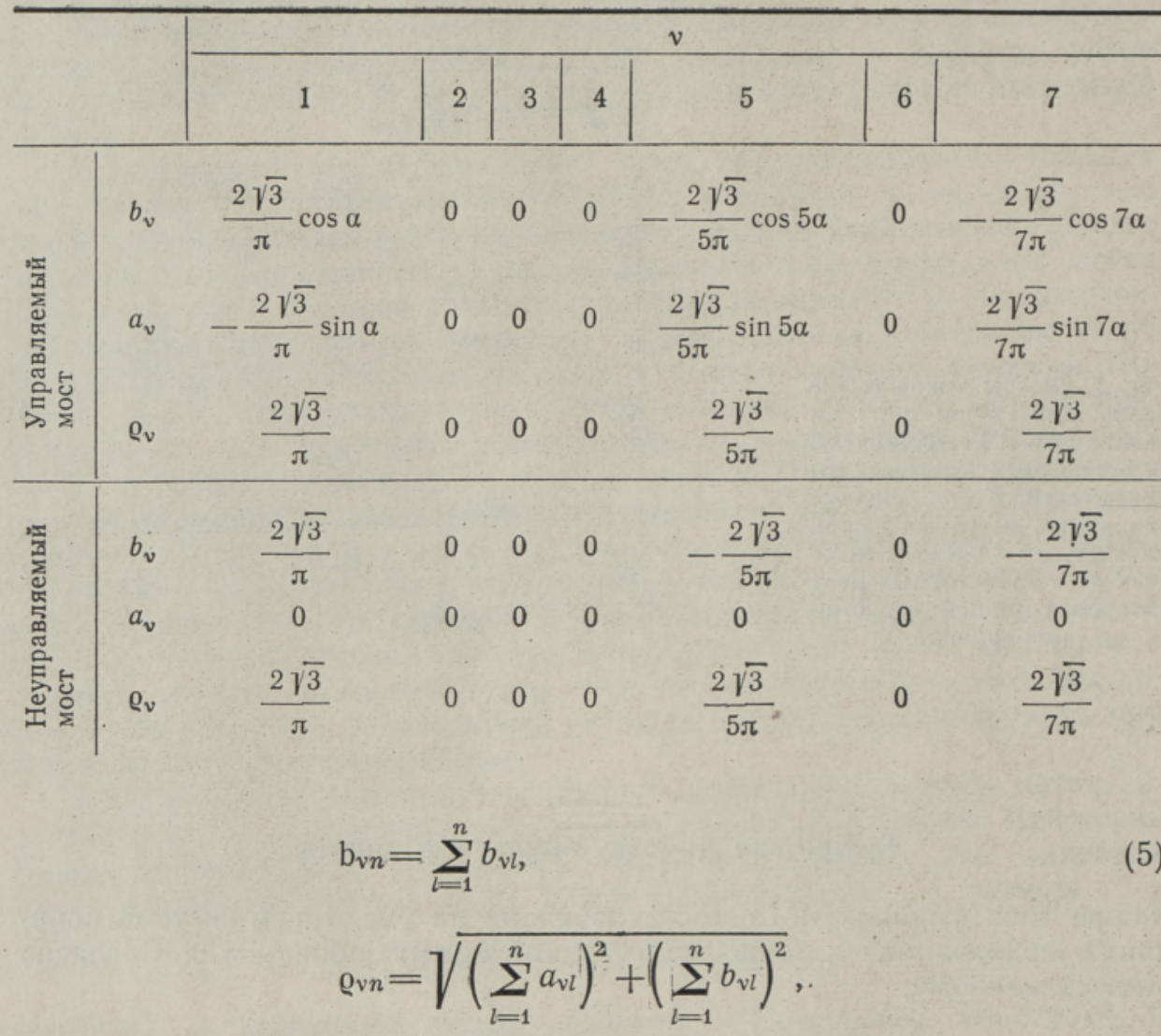

а коэффициент сдвига $\varphi_{v}=\sum_{l=1}^{n} b_{v l} / \sum_{l=1}^{n} a_{v l}$.

Найдем соотношение гармоник для суммарного тока

$$
\begin{aligned}
\zeta_{v n} & =\frac{\varrho_{v n}}{\varrho_{1 n}}=\frac{\sqrt{\left(-\frac{1}{v \pi}\left(2 \sqrt{3} \sum_{l=1}^{n} \cos v \alpha_{l}\right)\right)^{2}+\left(\frac{1}{v \pi}\left(2 \sqrt{3} \sum_{l=1}^{n} \sin v \alpha_{l}\right)\right)^{2}}}{\sqrt{\left(\frac{1}{\pi}\left(2 \sqrt{3} \sum_{l=1}^{n} \cos \alpha_{l}\right)\right)^{2}+\left(-\frac{1}{\pi}\left(2 \sqrt{3} \sum_{l=1}^{n} \sin \alpha_{l}\right)\right)^{2}}}= \\
& =\frac{1}{v} \sqrt{\frac{\left(\sum_{l=1}^{n} \cos v \alpha_{l}\right)^{2}+\left(\sum_{l=1}^{n} \sin v \alpha_{l}\right)^{2}}{\left(\sum_{l=1}^{n} \cos \alpha_{l}\right)^{2}+\left(\sum_{l=1}^{n} \sin \alpha_{l}\right)^{2}}} .
\end{aligned}
$$

Для равноинтервального управления (которое можем создать) $\alpha_{l+1}=$ $=\alpha_{l}+\Delta_{\alpha}$, что позволяет (6) преобразовать

$$
\zeta_{v n}=\frac{1}{v} \sqrt{\frac{\left(\sum_{l=1}^{n-1} \cos v(l-1) \Delta_{\alpha}\right)^{2}+\left(\sum_{l=1}^{n-1} \sin v(l-1) \Delta_{\alpha}\right)^{2}}{\left(\sum_{l=1}^{n-1} \cos (l-1) \Delta_{\alpha}\right)^{2}+\left(\sum_{l=1}^{n-1} \sin (l-1) \Delta_{\alpha}\right)^{2}}} .
$$




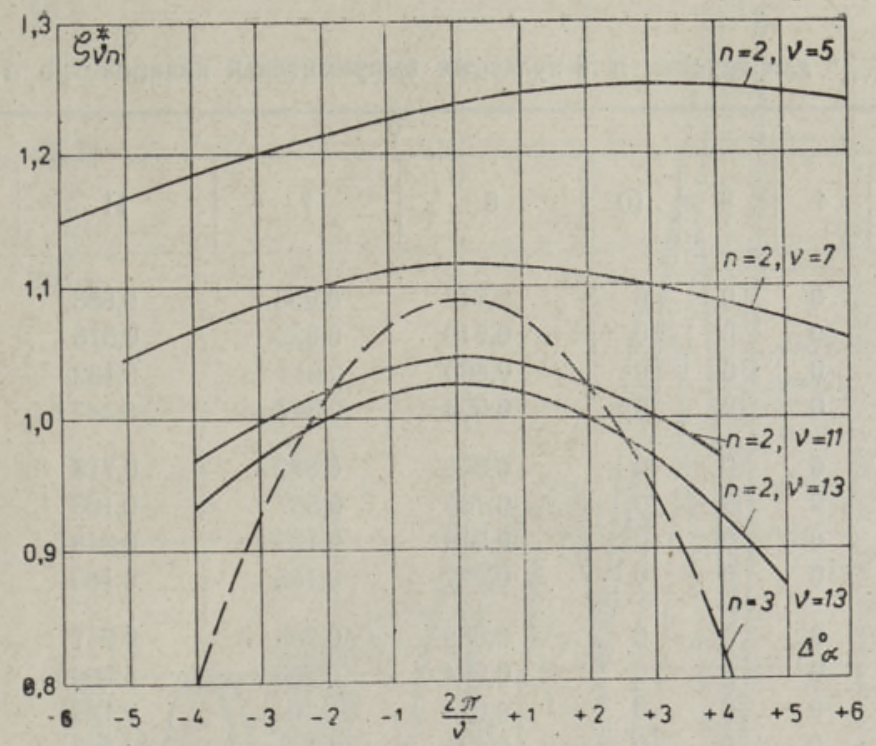

Рйс. 2. Әффект кумуляции высших гармонических составляющих в системе 6-пульсных мостовых выпрямителей.

Интересным параметром, характеризующим систему, является относительное соотношение высшей гармонической составляющей

$$
\zeta_{v n}^{*}=\frac{\zeta_{v n}}{\zeta_{v l}}=\sqrt{\frac{\left(\sum_{l=1}^{n-1} \cos v(l-1) \Delta_{\alpha}\right)^{2}+\left(\sum_{l=1}^{n-1} \sin v(l-1) \Delta_{\alpha}\right)^{2}}{\left(\sum_{l=1}^{n-1} \cos (l-1) \Delta_{\alpha}\right)^{2}+\left(\sum_{l=1}^{n-1} \sin (l-1) \Delta_{\alpha}\right)^{2}}}
$$

которое показывает подавление (повышение) высшей гармонической составляющей в системе, достигаемое расстройкой (смещением рабочей точки) управляемых выпрямителей. По (8) можно заключить, что в системе 6-пульсных выпрямителей указанное подавление $\zeta^{*}{ }_{v n} \neq f(\alpha)$ инвариантно углу управления. Многомерность $\zeta^{*}{ }_{v n}$ затрудняет его графическое изображение. Поэтому $\zeta^{*}{ }_{v n}\left(v, n, \Delta_{\alpha}\right)$ представлено в табл. 2 , где в каждой клетке показан столб из четырех чисел, полученных для различных равномерных расстроек $\Delta_{\alpha} \in\left\{5,10,15,20^{\circ}\right.$ эл. $\}$. По табл. 2 можно заключить, что при малых $n, v$ и $\Delta_{\alpha}$ в группе достигается подавление высших гармоник (низкого порядка), причем оно усугубляется с увеличением порядка гармоник, расстройки и числа входящих в группу выпрямителей. Однако эта закономерность оказывается несостоятельной при условии $\Delta_{\alpha} \rightarrow 2 \pi / v$, где в связи с подходящими фазовыми соотношениями наблюдается кумуляция высших гармонических составляющих. Амплитуда кумуляции уменьшается с ростом порядка гармоник и увеличивается с увеличением числа входящих в группу выпрямителей. Это показано на рис. 2 при изменении расстройки в области $\Delta_{\alpha} \approx 2 \pi / v$. Ограниченный диапазон регулирования по $\alpha$ не позволяет совместить одновременно большие расстройки и большие числа входящих в группу выпрямителей. Поэтому эффект кумуляции высших гармонических составляющих не приносит практического вреда. Следовательно, в системе 6-пульсных выпрямителей можно использовать преднамеренную расстройку выпрямителей в целях улучшения гармонического состава сум. марного тока. 
$\zeta_{v n}{ }^{*}$ для группы $n$ 6-пульсных выпрямителей инвариантно $\alpha$

\begin{tabular}{|c|c|c|c|c|c|c|c|c|}
\hline \multirow{2}{*}{$n$} & \multicolumn{8}{|c|}{$v$} \\
\hline & 2 & 4 & 8 & 10 & 5 & 7 & 11 & 13 \\
\hline \multirow{4}{*}{2} & 0 & 0 & 0 & 0 & 0,977 & 0,955 & 0,888 & 0,944 \\
\hline & 0 & 0 & 0 & 0 & 0,910 & 0,822 & 0,576 & 0,424 \\
\hline & 0 & 0 & 0 & 0 & 0,800 & 0,614 & 0,132 & 0,132 \\
\hline & 0 & 0 & 0 & 0 & 0,653 & 0,347 & 0,347 & 0,652 \\
\hline \multirow{4}{*}{3} & 0 & 0 & 0 & 0 & 0,940 & 0,882 & 0,718 & 0,617 \\
\hline & 0 & 0 & 0 & 0 & 0,769 & 0,567 & 0,106 & 0,962 \\
\hline & 0 & 0 & 0 & 0 & 0,518 & 0,165 & 0,318 & 0,318 \\
\hline & 0 & 0 & 0 & 0 & 0,227 & 0,185 & 0,185 & 0,220 \\
\hline \multirow{4}{*}{4} & 0 & 0 & 0 & 0 & 0,889 & 0,785 & 0,511 & 0,358 \\
\hline & 0 & 0 & 0 & 0 & 0,594 & 0,286 & 0,200 & 0,277 \\
\hline & 0 & 0 & 0 & 0 & 0,214 & 0,164 & 0,132 & 0,132 \\
\hline & 0 & 0 & 0 & 0 & 0,121 & 0,283 & 0,283 & 0,120 \\
\hline
\end{tabular}

\section{Групповая работа полууправляемых мостов}

По рис. 1,б, где изображена эпюра тока полууправляемого моста в системе координат, привязанной к точке естественной коммутации $\hat{\alpha}=0$, видно, что она может быть рассмотрена как сумма токов неуправляемого моста и помех и представлена в виде импульсного сигнала цля случая $\alpha>\pi / 3$ (рис. $1, \varepsilon$ ). Коэффициенты разложения Фурье тока для неуправляемого выпрямителя были найдены выше. Следовательно, здесь достаточно найти соответствующие коэффициенты указанной помехи

$$
\begin{aligned}
& a_{v}=\frac{1}{\pi}\left[\int_{\pi}^{\pi+\alpha}(-\cos v \omega t) d \omega t+\int_{5 \pi / 3}^{5 \pi / 3+\alpha} \cos v \omega t d \omega t\right], \\
& b_{v}=\frac{1}{\pi}\left[\int_{\pi}^{\pi+\alpha}(-\sin v \omega t) d \omega t+\int_{5 \pi / 3}^{5 \pi / 3+\alpha} \sin v \omega t d \omega t\right] .
\end{aligned}
$$

Вычисленные по (9) и табл. 1 коэффициенты разложения Фурье и амплитуды тока неуправляемого выпрямителя могут быть представлены в аналитическом виде следующим образом.

Для нечетных гармоник $v \in\{1,5,7,11,13\}$

$$
\begin{aligned}
& a_{v}=(1 / 2 v \pi)(\mp \sqrt{3}(1+\cos v+3 \sin v \alpha)), \\
& b_{v}=(1 / 2 v \pi)(-3(1+\cos v \alpha) \mp \sqrt{3} \sin v \alpha), \\
& \varrho v=(\sqrt{6} / v \pi) \sqrt{1+\cos \alpha}
\end{aligned}
$$

и для четных $v \in\{2,4,8,10\}$

$$
\begin{aligned}
& a_{v}=(1 / 2 v \pi)( \pm \sqrt{3}(1-\cos v \alpha)-3 \sin v \alpha), \\
& b_{v}=(1 / 2 v \pi)(-\sqrt{3}(1-\cos v \alpha) \mp 3 \sin v \alpha),
\end{aligned}
$$




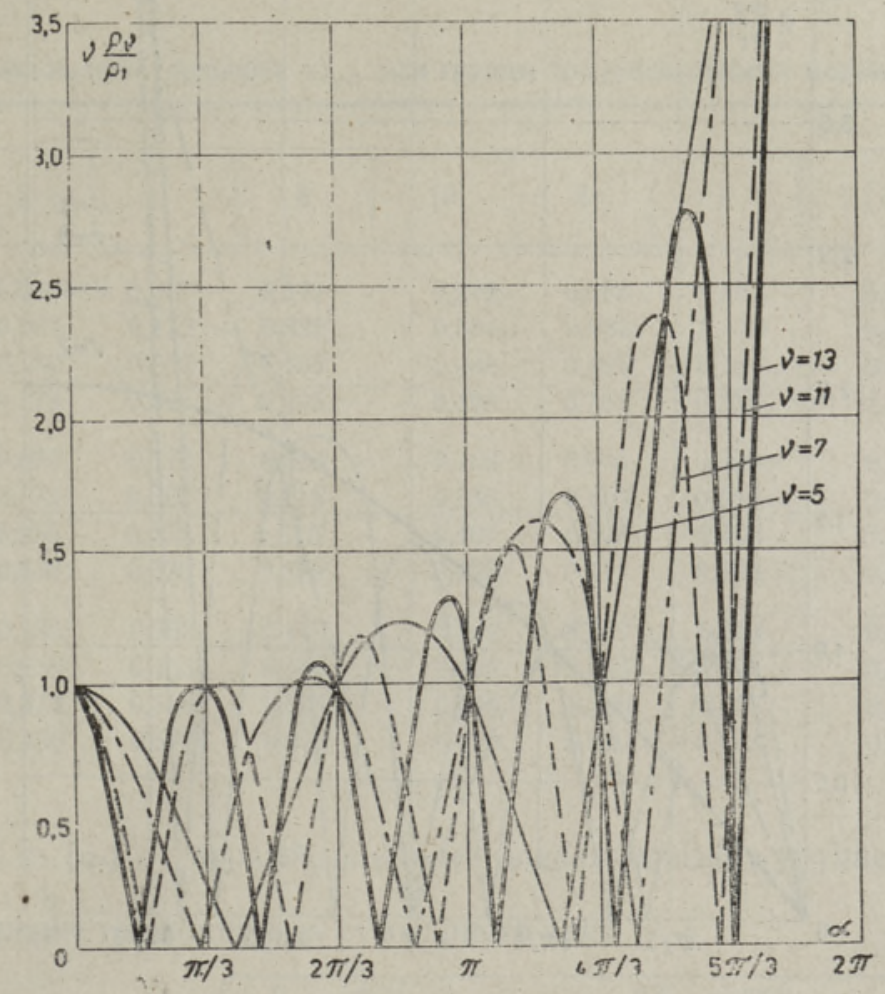

Рис. 3. Нормализованные амплитуды нечетных гармоник входного тока полууправляемого моста.

$$
\dot{\varrho}=(\sqrt{6} / v \pi) \sqrt{1-\cos v \alpha} .
$$

Знаки плюс и минус в формулах (10) и (11) чередуются согласно указанной очередности гармоник. Видно, что полууправляемый мостовой выпрямитель обладает богатым спектром входного тока, в котором отсутствуют лишь гармоники, кратные трем $v=\{3,6,9,12 \ldots\}$. В отличие от 6-пульсного выпрямителя в данном случае амплитуды всех гармоник зависимы от угла управления $\mathrm{v}(\alpha) \neq$ const. Следовательно, не может быть инвариантным относительно угла управления и соотношение амплитуды гармоники высшего порядка с амплитудой первой гармоники

$$
\begin{aligned}
& \zeta_{v}=\frac{\varrho v}{\varrho_{1}}=\frac{1}{v} \sqrt{\frac{1+\cos v \alpha}{1+\cos \alpha}} \text { для нечетных и } \\
& \zeta_{v}=\frac{1}{v} \sqrt{\frac{1-\cos v \alpha}{1+\cos \alpha}} \text { для четных гармоник. }
\end{aligned}
$$

Графики нормализованных функций $v \zeta_{v}$ на рис. 3 для нечетных и на рис. 4 для четных гармоник показывают, что с помощью режима выпрямителя можем подобрать такую $\alpha$, при которой нежелаемая гармоника (высших порядков $v \leqslant 4$ ) будет иметь нулевую амплитуду. Однако это можно сделать только по одной из гармоник, так как нельзя достичь режима, одновременно благоприятного для подавления всех присутствующих гармоник. Углы управления, обеспечивающие нулевую амплитуду, могут быть найдены по (12). Для нечетных

$$
\alpha\left(\varrho_{v}=0\right)=2 N \pi / v \leqslant \pi
$$




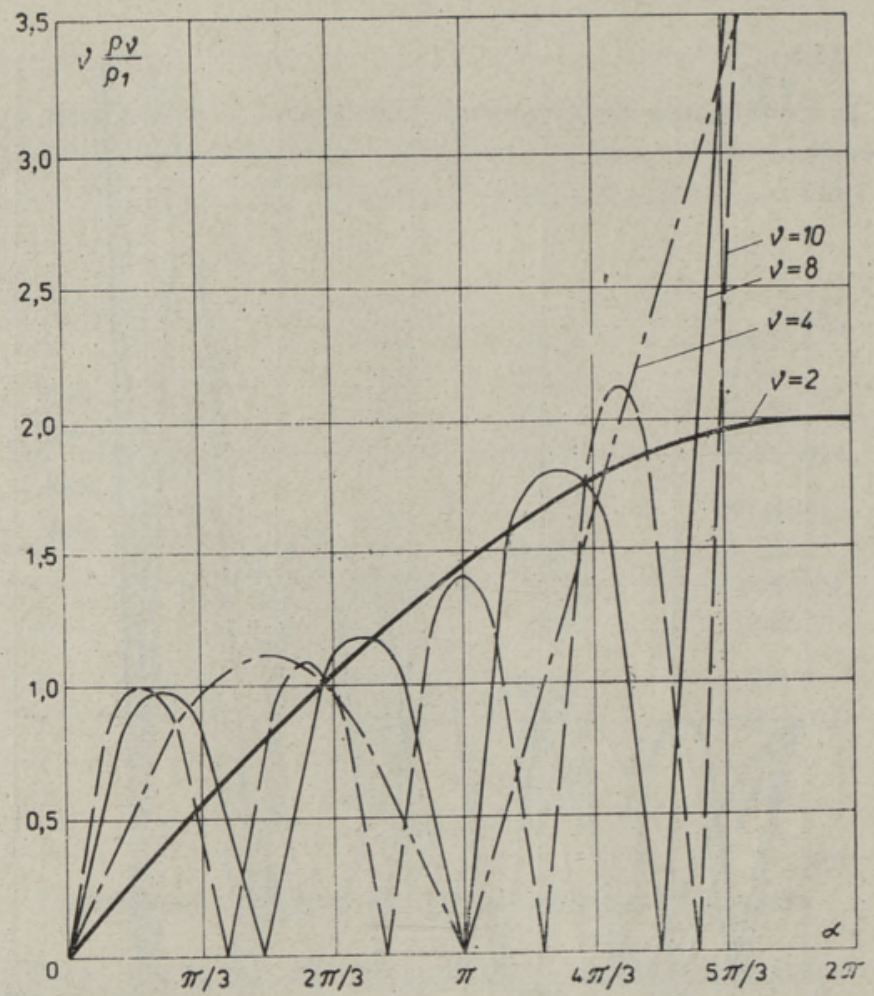

Рис. 4. Нормализованные амплитуды четных гармоник входного тока полууправляемого моста.

и для четных

$$
\alpha(\varrho v=0)=(2 N-1) \pi / v \leqslant \pi,
$$

где $N \in\{N\}$.

Коэффициенты Фурье суммарного тока группы полууправляемых выпрямителей могут быть найдены по (5), если подставить частные коэффициенты из (10) и (11). Приведем здесь лишь амплитуды гармоник. Для нечетных и четных гармоник

$$
\begin{aligned}
& \varrho v=\frac{1}{2 v \pi} \sqrt{9\left(\sum_{l=1}^{n}\left(1 \pm \cos v \alpha_{l}\right)\right)^{2}+3\left(\sum_{l=1}^{n}\left(1+\cos v \alpha_{l}\right)\right)^{2}}+ \\
& \overline{+9\left(\sum_{l=1}^{n} \sin v \alpha_{l}\right)^{2}+3\left(\sum_{l=1}^{n} \sin v \alpha_{l}\right)^{2}}= \\
& =\frac{\sqrt{3}}{v \pi} \sqrt{\left(\sum_{l=1}^{n}\left(1 \pm \cos v \alpha_{l}\right)\right)^{2}+\left(\sum_{l=1}^{n} \sin v \alpha_{l}\right)^{2}}
\end{aligned}
$$

причем знак минус относится к четным, а знак плюс - к нечетным гармоникам. Для равномерного распределения расстройки углов управления

$$
\varrho v=\frac{\sqrt{3}}{v \pi} \sqrt{\left(n \pm \sum_{l=0}^{n-1} \cos v\left(\alpha_{1}+l \Delta_{\alpha}\right)\right)^{2}+\left(\sum_{l=0}^{n-1} \sin v\left(\alpha_{1}+l \Delta_{\alpha}\right)\right)^{2}}
$$


Нормализованная функция $v \zeta_{v n}$ для группы полууправляемых мостов при $\alpha_{\mathrm{r}}=0$

\begin{tabular}{|c|c|c|c|c|c|c|c|c|}
\hline \multirow{2}{*}{$n$} & \multicolumn{7}{|c|}{$v$} & \multirow[t]{2}{*}{ - } \\
\hline & 2 & 4 & 8 & 10 & 5 & 7 & 11 & \\
\hline \multirow{4}{*}{2} & 0,043 & 0,087 & 0,171 & 0,212 & 0,983 & 0,966 & 0,917 & 0,886 \\
\hline & 0,087 & 0,172 . & 0,322 & 0,384 & 0,933 & 0,870 & 0,707 & 0,621 \\
\hline & 0,130 & 0,252 & 0,436 & 0,486 & 0,855 & 0,731 & 0,516 & 0,516 \\
\hline & 0,173 & 0,325 & 0,498 & 0,498 & 0,757 & 0,588 & 0,588 & 0,757 \\
\hline \multirow{4}{*}{3} & 0,087 & 0,172 & 0,324 & 0,389 & 0,948 & 0,898 . & 0,765 & 0,690 \\
\hline & 0,173 & 0,327 & 0,516 & 0,535 & 0,807 & 0,658 & 0,489 & 0,537 \\
\hline & 0,256 & 0,450 & 0,510 & 0,407 & 0,628 & 0,495 & 0,664 & 0,664 \\
\hline & 0,336 & 0,530 & 0,379 & 0,379 & 0,511 & 0,592 & 0,592 & 0,511 \\
\hline \multirow{4}{*}{4} & 0,130 & 0,253 & 0,449 & 0,512 & 0,897 & 0,804 & 0,593 & 0,511 \\
\hline & 0,256 & 0,455 & 0,546 & 0,461 & 0,652 & 0,492 & 0,606 & 0,644 \\
\hline & 0,374 & 0,568 & 0,391 & 0,446 & 0,490 & 0,597 & 0,549 & 0,549 \\
\hline & 0,480 & 0,577 & 0,499 & 0,499 & 0,590 & 0,663 & 0,663 & 0,590 \\
\hline
\end{tabular}

Здесь $\alpha_{1} \in\left\{\alpha_{l}\right\}-$ первый, наиболее опережающий в группе угол управления.

Соотношение гармоник для суммарного тока

$$
\zeta_{v n}=\frac{1}{v} \sqrt{\frac{\left(n \pm \sum_{l=0}^{n-1} \cos v\left(\alpha_{1}+l \Delta_{\alpha}\right)\right)^{2}+\left(\sum_{l=0}^{n-1} \sin v\left(\alpha_{1}+l \Delta_{\alpha}\right)\right)^{2}}{\left(n+\sum_{l=0}^{n-1} \cos \left(\alpha_{1}+l \Delta_{\alpha}\right)\right)^{2}+\left(\sum_{l=0}^{n-1} \sin \left(\alpha_{1}+l \Delta_{\alpha}\right)\right)^{2}}} .
$$

Видно, что $\xi_{v n}=f\left(n, v, \alpha, \Delta_{\alpha}\right)$ является четырехмерной функцией, которая может быть охарактеризована пакетом таблиц [ $\left.{ }^{16}\right]$. С целью иллюстрации полученных результатов в табл. 3 представлена нормализованная функция $v \zeta_{v n}$ для $\alpha_{1}=0$. Анализ этой функции в окрестности точек $\alpha \rightarrow 2 \pi / v$ показывает также наличие эффекта кумуляции высших гармоник, который, однако, слабее соответствующего эффекта при работе 6-пульсных выпрямителей, и поэтому ему больше внимания не уделяется.

Соответственно, пакетом таблиц может быть представлено и относительное соотношение $\zeta^{*}{ }_{v n}=f\left(n, v, \alpha_{1}, \Delta_{\alpha}\right)$, которое для сравнения приведено в табл. 4 для $\alpha_{1}=0$. Относительно последнего (наглядного показателя) следует сделать два замечания. 1) Согласно (10), Q1 есть зависимая от $\alpha$ величина. При расстройке углов управления сравнение следует проводить с единичным полууправляемым выпрямителем с усредненным углом управления

$$
\bar{\alpha}=\alpha_{1}+\frac{(n-1) \Delta_{\alpha}}{2} .
$$

2) Согласно (12), $\zeta_{v}=0$ в точках (13). Следовательно, в этих точках $\zeta^{*}{ }_{v n} \rightarrow \infty$, что является бессмысленным. Другими словами, для оценки эффекта подавления высших гармонических при групповой работе полууправляемых выпрямителей следовало либо разработать новую интегральную оценку, либо очистить $\zeta^{*}$ vn от значений, полученных вблизи 
$\zeta_{v n}{ }^{*}$ для группы $n$ полууправляемых мостовых выпрямителей при $\alpha_{1}=0$

\begin{tabular}{|c|c|c|c|c|c|c|c|c|}
\hline \multirow{2}{*}{$n$} & \multicolumn{8}{|c|}{$\bar{v}$} \\
\hline & 2 & 4 & 8 & 10 & 5 & 7 & 11 & 13 \\
\hline \multirow{4}{*}{2} & 0,999 & 0,997 & 0,985 & 0,977 & 0,989 & 0,977 & 0,944 & 0,922 \\
\hline & 0,998 & 0,987 & 0,942 & 0,908 & 0,955 & 0,912 & 0,796 & 0,736 \\
\hline & 0,996 & 0,970 & 0,870 & 0,797 & 0,901 & 0,814 & 0,685 & 0,781 \\
\hline & 0,992 & 0,947 & 0,772 & 0,648 & 0,832 & 0,715 & 1,021 & - \\
\hline \multirow{4}{*}{3} & 0,998 & 0,988 & 0,948 & 0,918 & 0,970 & 0,941 & 0,862 & 0,817 \\
\hline & 0,992 & 0,951 & 0,800 & 0,695 & 0,887 & 0,799 & 0,849 & 1,265 \\
\hline & 0,981 & 0,892 & 0,584 & 0,418 & 0,785 & 0,807 & - & - \\
\hline & 0,966 & 0,812 & 0,379 & 0,379 & 0,783 & - & - & \\
\hline \multirow{4}{*}{4} & 0,996 & 0,975 & 0,896 & 0,839 & 0,945 & 0,894 & 0,786 & 0,773 \\
\hline & 0,982 & 0,902 & 0,625 & 0,473 & 0,815 & 0,801 & - & - \\
\hline & 0,960 & 0,787 & 0,383 & 0,473 & 0,865 & - & 0,970 & 0,648 \\
\hline & 0,928 & 0,643 & 0,557 & 0,965 & - & - & 0,663 & 0,590 \\
\hline
\end{tabular}

аргумента, удовлетворяющего (13). Именно второй, упрощенный метод выбран при представлении табл. 4 и таблиц в $\left[{ }^{16}\right]$. Расчетный алгоритм табл. 4 с учетом (17) получается в виде

$$
\xi_{v n}^{*}=\sqrt{\frac{(1+\cos \bar{\alpha})\left[n \pm\left(\sum_{l=0}^{n-1} \cos v\left(\alpha_{1}+l_{\Delta \alpha}\right)\right)^{2}+\left(\sum_{l=0}^{n-1} \sin v\left(\alpha_{1}+l \Delta_{\alpha}\right)\right)^{2}\right]}{(1 \pm \cos v \bar{\alpha})\left[n+\left(\sum_{l=0}^{n-1} \cos \left(\alpha_{1}+\bar{l} \Delta_{\alpha}\right)\right)^{2}+\left(\sum_{l=0}^{n-1} \sin \left(\alpha_{1}+l \Delta_{\alpha}\right)\right)^{2}\right]}}
$$

для нечетных и четных гармоник соответственно. Сравнение табл. 4 и табл. 2 показывает, что подавление высших гармонических при групповой работе полууправляемых выпрямителей проявляется слабее, чем соответствующее подавление при групповой работе 6-пульсных выпрямителей. В первом приближении при малых расстройках $\Delta_{\alpha}<5^{\circ}$ эл. и им можно пренебречь и считать, что суммарный ток системы выпрямителей с общей диодной частью описывается как ток полууправляемого выпрямителя с суммарной мощностью и усредненным углом управления. Для гармоник низких порядков $v \in\{2,4,5,7\}$ получаем при этом оценку сверху с точностью $10-20 \%$.

\section{Тиристорные регуляторы с общей группой тиристоров}

Проанализируем входной ток устройства $\left[{ }^{17}\right]$, который состоит из $n$ самостоятельных трехтиристорных стабилизаторов. постоянного тока и общей для них тиристорной группы, управляемой пакетом импульсов, формированного посредством сумматора от управляющих импульсов всех $n$ регуляторов. Предположим, что работа устройства происходит в зоне $\alpha<\pi / 3$ и нет необходимости обращать внимания на спешифические ограничения, связанные с управлением общей тиристорной группой. В таком случае эпюра токов приобретает вид, изображенный на рис. 1,2 для системы при $n=2$. Первый регулятор с опережающим углом $\alpha^{\prime}$ работает в режиме управляемого 6-пульсного моста со спектром, показанным в табл. 1. Все остальные $n-1$ регуляторы работают как не- 
управляемые мосты с относительным углом управления $\alpha_{l}{ }^{*}=\alpha_{l}-\alpha^{\prime}$, т. е. для них действительны формулы (10)-(17) и заключения относительно групповой работы полууправляемых мостовых выпрямителей. Следует иметь в виду, что все гармонические, не присутствующие в 6-пульсном выпрямителе, а также составляющие гармоник $w \in$ $\in\{5,7,11,13\}$ за счет запаздывающих регуляторов, найдены с фазовым сдвигом $v \alpha_{1}$ относительно гармоник опережающего регулятора. Это необходимо учитывать при расчете спектра суммарного тока. Поскольку $\alpha_{l}{ }^{*}<\alpha_{l}$, а зачастую (для симметричных нагрузок) $\alpha_{l}{ }^{*} \rightarrow 0$, то согласно табл. 3 , достигается значительное уменьшение амплитуд четных гармоник.

\section{Выводы}

В случае групповой работы 6-пульсных выпрямителей при расстройке углов управления происходит подавление (частичная компенсация) гармоник невысокого порядка. Это подавление усугубляется с увеличением группы и порядка гармоник, однако если расстройка углов управления достигает определенной величины, наблюдается кумуляция гармоник. Эти эффекты оба присущи групповой работе полууправляемых мостов, но проявляются в меньшей мере по сравнению с групповой работой 6-пульсных выпрямителей. Прнменение тиристорных регуляторов с общей тиристорной группой - эффективный способ улучшения спектра употребляемого из сети тока для системы выпрямителей $\mathfrak{c}$ общей частью.

\section{ЛИТЕРАТ У РА}

1. Тамкиви П., Томсон Т. Изв. АН ЭССР. Физ. Матем., 31, № 4, 454-457 (1982).

2. Тамкиви П. И., Томсон $Т$. И. В кн.: Тепло- и массообмен в плазмохимических процессах, ч. 1. Минск, АН БССР, 1982.

3. Крайчик Ю. С. В кн.: Передача энергии постоянным и переменным током. Л., Изв. НИИПТ, 14, 70-75 (1968).

4. Анисимов Я. Ф. Изв. вузов СССР. Энергетика, № 9, 42-47 (1974).

5. Анисимов Я. Ф. Тр. НКИ, вып. 87, 14-19 (1974).

6. Нижин В. Н., Легостаев Н. С. В кн: Магнитно-вентиль"ые преобразователи параметров электрической энергии. Томск, Изд. Томск. ГУ, 1977, 88-94.

7. Pelly, B. R. Thyristor Phase-Controlled Converters and Cycloconverters. New York, 1971.

8. Червӧнекис Я. М. Изв. АН СССР. Отделение техн. н., № 4, 449-457 (1948).

9. Крогерис А. Ф. Изв. АН ЛатвССР, сер. физ. и техн. Н. № 7, 91-111 (1954).

10. Крогерис А. Ф. В кн.: Вопросы энергетики, вып. 3. Рига, Изд. АН ЛатвССР, 1955, $23-42$.

11. Поссе А. В. В кн.: Пепелача энергии постоянным и переменным током. Л., Изв. НИиПТ, 9, 46-62 (1962).

12. Крайчик Ю. С., Пинцов А. М., Слепокурова А. С., Тетерин В. П. В кн.: Передача энергии постоянным и переменным током. Л., Изв. НИИПТ, 16, 199-307 (19.70).

13. Глинтерник C. Р. Электромагнитные процессы и режимы мощных статистических преобразователей. Л., «Наука», 1968.

14. Полупроводниковые выпрямители (под ред. Ф. И. Ковалева и Г. П. Мостковой) М., «Энергия», 1978.

15. Крайчик FO. С. В кн.: Передача энергии постоянным н переменным током. Л., Изв. НИиПт, 16, 26-28 (1970).

16. Драгунов В., Томсон T. Рукопись депонирована в ВИНИТИ.

17. Калмо Т. Я., Тамкиви П. И., Томсон Т. И. Авт. свид. СССР № 1008865, 3/51/HО2М 7/12 БИ, № 12 (1983). 


\section{JUHITAVATE ALALDITE GRUPI SISENDVOOLU SPEKTER}

On uuritud $h$ juhitava alaldi baasil ehitatud voolugeneraatorite grupi summaarset sisendvoolu. Voolugeneraatori eritunnuseks on voolu jääv suurus alaldi juhtimisnurgast sõltumata. See lubab voolu aproksimeerida normaliseeritud nelinurksignaaliga. 6-pulsiliste alaldite grupi puhul on pööratud tähelepanu suhtele $\zeta^{*}{ }_{v n}$, mis näitab summaarses voolus sisalduva kõrgema harmoonilise suhtelist amplituudi, vōrreldes sama võimsusega üksiku alaldi vastava amplituudiga. Nimetatud tegur näitab, et sellises grupis saavutatakse juhtimisnurkade lahkuhäälestusest tingituna summıarse voolu parem spektraalne koostis. Paranemist piirab kōrgemate harmooniliste kumulatsiooni efekt, mis esineb ühtivatest faasivahekordadest tingituna lahkuhäälestuse $\cdot$ väärtuse $\Delta_{\alpha} \approx 2 \pi / v$ ümbruses. Pooljuhtalaldite grupi puhul esinevad juhtimisnurgad, mille puhul mōne kõrgema harmoonilise amplituud vôib olla väärtusega 0 . See piirab $\zeta^{*}{ }_{v}^{n}$ kasutamispiirkonda, kuid analüüsi tulemustest selgub, et pooljuhtalaldite grupi puhul on nii signaali spektri paranemise kui ka kõrgemate harmooniliste kumulatsiooni efekt vähem väljendatud.

\section{T. TOMSON}

\section{THE INPUT CURRENT SPECTRUM OF A CONTROLLED RECTIFIER GROUP}

The total spectrum of input current in a group of controlled rectifiers has been analyzed. Every rectifier operates in the current generator conditions that enable to approximate any separate input current with a normalized square signal independent of the firing angle. In case of a fully controlled rectifier group the relation $\zeta^{*}{ }^{n}$ shows the relative amplitude of any $v-$ harmonic in the total current compared with the current of a single (equal power) rectifier. This relation shows that the current in this group is more sinusoidal than in a single rectifier because of the different firing angle. This kind of filtration of the input current is limited by the influence of the harmonic accumulation based on a certain phase relation around the dissimilarity of angles $\Delta_{\alpha} \approx 2 \pi / v$.

In the group of half-controlled rectifiers the harmonic amplitudes have zero values at some firing angles.

It limits the ranged use of $\zeta^{*}{ }_{v^{n}}$, but it can be concluded from the results of the analyses that both the filtration and accumulation effects are less expressed for this group. 